\title{
Spontaneous superradiant sub-THz coherent cyclotron emission from a short dense electron bunch
}

\author{
Yu. S. Oparina ${ }^{1}$ and A. V. Savilov ${ }^{1,2}$ \\ ${ }^{1}$ Institute of Applied Physics, Russian Academy of Sciences, Nizhny Novgorod 603950 Russia \\ ${ }^{2}$ Lobachevsky State University of Nizhny Novgorod, Nizhny Novgorod 603950, Russia
}

(Received 28 May 2018; published 5 March 2019)

\begin{abstract}
Short dense electron bunches produced by modern photoinjectors are attractive from the viewpoint of the realization of powerful and effective sources of subterahertz radiation based on the spontaneous coherent mechanism of emission. This type of emission is realized if the effective phase size of the bunch with respect to the radiated wave is small enough. Therefore, the repulsion of particles caused by a strong Coulomb field inside the dense electron bunch strictly limits the duration of the radiation process due to the increase in the bunch length. We show that this problem can be solved by using the cyclotron mechanism of the spontaneous radiation due to the effect of compensation of the Coulomb repulsion in the phase space.
\end{abstract}

DOI: 10.1103/PhysRevAccelBeams.22.030701

\section{INTRODUCTION}

Modern sources of dense electron beams (including laser-driven photoinjectors) allow the formation of compact and accessible sources of dense electron bunches with a moderate energy of 3-6 MeV, picosecond pulse durations, and charges of up to $1 \mathrm{nC}$ and even greater [1-6]. These bunches can be used for the realization of relatively simple and compact terahertz $(\mathrm{THz})$ sources operating in the regime of spontaneous coherent radiation [7-14]. This type of radiation is realized when the effective phase size of the electron bunch with respect to the wave is small enough so that the wave packets emitted by each of the electrons add up basically in phase (Fig. 1). In other words, in this paper, spontaneous emission means a situation where the electronic bunch is so short that it is ideally (or almost ideally) bunched with respect to the emitted wave already at the entrance to the radiation region and remains so during the whole process of radiation. In the case of undulator radiation, this situation is provided when the bunch stays significantly shorter than the wavelength of the radiated wave $[13,14]$. In the case of cyclotron radiation, the situation is more complicated due to the $2 \mathrm{D}$ character of the electron phase with respect to the wave. This paper is devoted to the peculiarities of providing and maintaining the spontaneous nature of the cyclotron radiation from a short electron bunch.

Published by the American Physical Society under the terms of the Creative Commons Attribution 4.0 International license. Further distribution of this work must maintain attribution to the author(s) and the published article's title, journal citation, and DOI.
Sub-THz and THz sources based on spontaneous emission have a number of advantages as compared to the more traditional electron masers based on the emission induced due to the bunching of a long electron beam by the radiated wave [Fig. 1(b)]. First of all, an evident advantage is a relatively high efficiency of the energy extraction from electrons, which can be achieved in a simple microwave system based on the "ready-for-radiation" bunch. Actually, such an oscillator does not require either a wave feedback system or an input wave signal to provide the highefficiency stimulated character of the radiation process. A high efficiency together with a narrow frequency band of the radiated rf signal is provided in a relatively short and simple system (namely, just an amplifierlike waveguide system). One more important advantage is that the phase of the radiated rf signal is fixed by the electron bunch phase.

A key problem in the realization of a sub- $\mathrm{THz}$ source based on the spontaneous coherent emission from a short electron bunch is a strong Coulomb repulsion in dense bunches, which leads to the increase of the axial bunch length [Fig. 1(a)]. If the "operating" radiation mechanism of the sub-THz source is based on the longitudinal electron bunching [15] (free-electron lasers and Cherenkov masers), then axial expansion of the bunch leads automatically to an increase in the bunch phase size with respect to the radiated wave. This results in the saturation of the process of spontaneous emission. Thus, in the case of undulator and Cherenkov masers, special methods providing control of the axial length of the bunch $[13,14]$ (and, possibly, the compression of the bunch [16-18]) are required.

This paper is devoted to studying possibilities for organizing spontaneous coherent emission from a short electron bunch in cyclotron masers. We develop the theory 

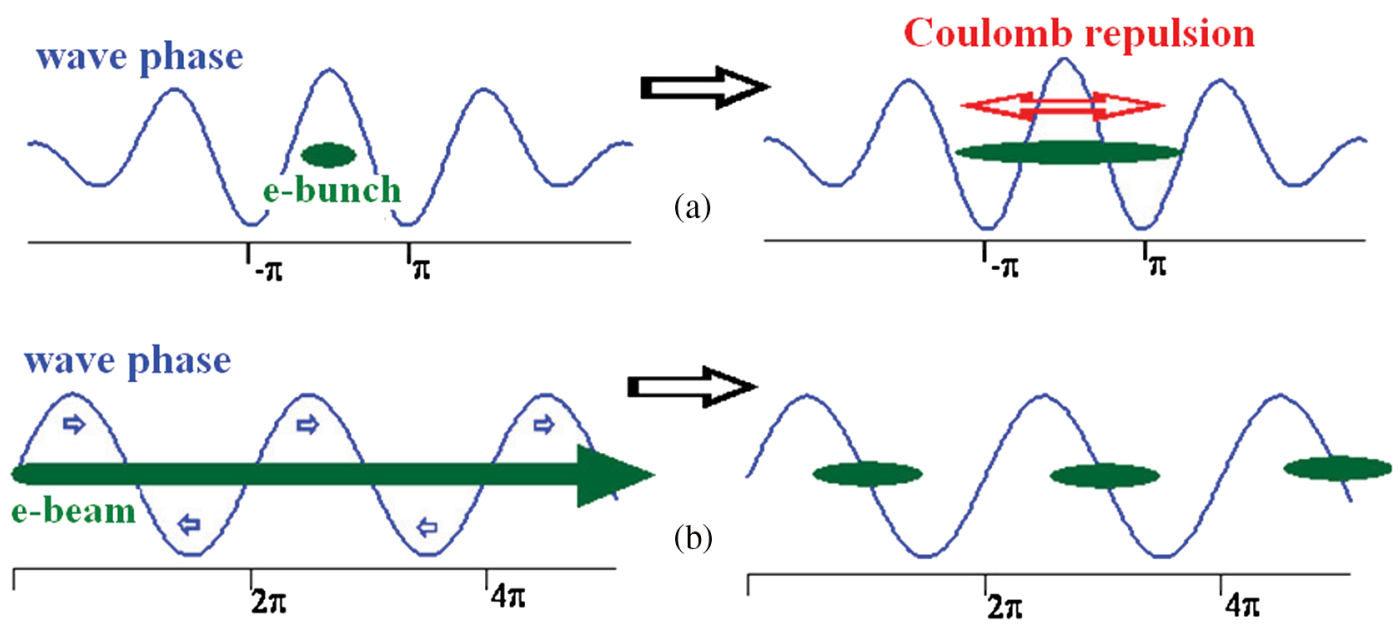

FIG. 1. (a) Spontaneous emission from a short electron bunch, as well as the termination of this process due to the increase in the phase size of the bunch caused by Coulomb repulsion. (b) Induced emission from a long electron beam of a traditional electron maser, which is based on the bunching of the beam in the field of the radiated wave.

of these devices [19-24] to a special case of spontaneous emission of a short wave packet from a short (shorter than the radiation wavelength) and dense electron bunch. Naturally, in this situation, the axial expansion of the bunch due to a strong Coulomb repulsion is very important for maintaining the spontaneous character of the emission. We show that an advantage of the cyclotron character of the spontaneous emission is that one can easily solve the problem of the Coulomb repulsion. Namely, it is possible to provide a situation where an increase in the axial length of the bunch caused by the Coulomb repulsion does not lead to an increase in the phase size of the bunch, so that the cyclotron phases of particles with respect to the radiated wave stay almost constant.

We show that this effect of compensation of the Coulomb repulsion in the phase space is provided automatically in the group resonance regime, where the group velocity of the radiated wave is close to the axial electron velocity and is useful also because the group resonance regime is very attractive from the viewpoint of organizing the wave emission process, as the maximal growth rate of the cyclotron instability is achieved in this regime [21]. The group resonance regime is especially important for short electron bunches, as in this situation a superradiant regime is realized $[25,26]$. In this regime, the radiated wave does not "run away" from the electron bunch, as the group velocity of the radiated wave packet coincides with the translational velocity of the electron bunch. Therefore, during the radiation process, the wave field is accumulated in the region close to the bunch. This accumulation leads to the formation of a powerful short wave pulse propagating together with the bunch. It was shown $[21,25,26]$ that this superradiant regime provides the maximal growth rate of the cyclotron instability in the case of the traditional induced character of the emission (when the bunch is much longer than the wavelength). In this paper, we use the advantages of this regime for the case of spontaneous emission from a short bunch.

This paper is organized as follows. The effect of compensation of the Coulomb repulsion in the space of cyclotron phases is described in Sec. II. In Sec. III, a set of equations describing electron motion in the radiated wave field and in the Coulomb field of the bunch is obtained. In Sec. IV, we study possibilities for generating short powerful wave pulses in an electron oscillator based on spontaneous coherent cyclotron emission. In Sec. V, we discuss some peculiarities of the regime of the spontaneous coherent superradiant emission.

\section{COMPENSATION OF THE COULOMB REPULSION IN THE SPACE OF CYCLOTRON PHASES}

In this paper, we consider the spontaneous cyclotron emission from a short dense electron bunch possessing parameters typical for modern photoinjectors (energy at the level of several $\mathrm{MeV}$ and charge at the $\mathrm{nC}$ level). A short (few picoseconds) duration of this bunch makes it possible to provide the spontaneous character of the emission in the sub- $\mathrm{THz}$ frequency range.

We assume that the bunch moves along a helix in a waveguide immersed in a uniform axial magnetic field (Fig. 2) and radiates a waveguide mode with a fixed transverse structure. In the simplest case, the transverse mode selection can be provided by excitation of the lowest mode in the regime of grazing of the dispersion characteristic, when the axial electron velocity coincides with the wave group velocity [Fig. 2(c)]. We show further that this regime is optimal also from the viewpoints of both the efficiency of the wave emission and compensation of the Coulomb repulsion of electrons in the space of their cyclotron phases. 

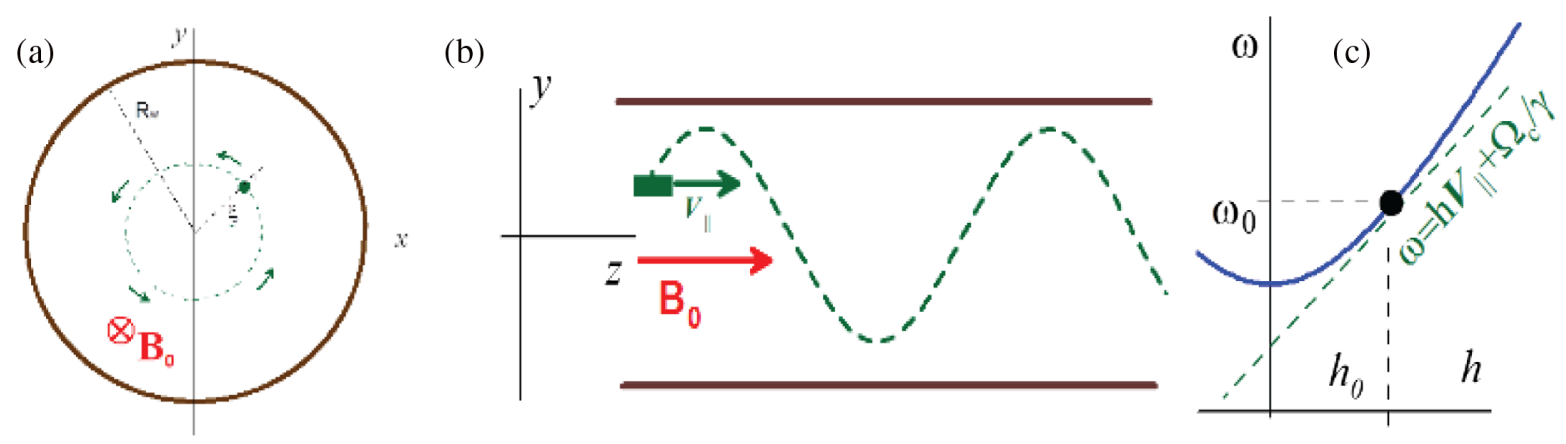

FIG. 2. (a) Cross section of the cavity. (b) Longitudinal section of the cavity. (c) Dispersion characteristic for the case when the electron velocity is close to the group velocity of the radiated wave.

We study the spontaneous coherent cyclotron emission from the short bunch. Such a type of emission takes place when the effective phase size of the bunch with respect to the radiated wave is small enough $(<2 \pi)$, so that almost all particles of the bunch radiate in phase. If electrons rotate around the waveguide axis (Fig. 1) and radiate the lowest circular-polarized wave $\mathrm{TE}_{1,1}$, then the electron phase with respect to the wave is determined as follows:

$$
\vartheta=\omega_{0} t-h_{0} z-\varphi
$$

Here, $\omega_{0}$ and $h_{0}$ are, respectively, the wave frequency and the axial wave number corresponding to the exact cyclotron resonance:

$$
\omega_{0}=h_{0} V_{\|}+\frac{\Omega_{c}}{\gamma} .
$$

In Eq. (2), $\varphi=\varphi_{0}+\int \Omega_{c} / \gamma d t$ is the phase of the electron gyrorotation determined by the relativistic electron cyclotron frequency $\Omega_{c} / \gamma$ (here, frequency $\Omega_{c}=\frac{e B_{0}}{m c}$ is the nonrelativistic cyclotron frequency, and $\gamma=1 / \sqrt{1-\beta^{2}}$ the relativistic Lorentz factor).

We consider the situation when electrons acquire gyrooscillations in a short kicker located at the entrance to the radiation space. In the approximation of a sufficiently short beam, we can assume that the initial gyrophases $\varphi_{0}$ are the same for all particles. Then, the initial electron phase with respect to the radiated wave is determined by the time the electron enters into the operating region:

$$
\vartheta_{0}=\omega_{0} t_{0} .
$$

Therefore, at the beginning of the electron-wave interaction region, initial cyclotron phases of all electrons in the bunch with respect to the radiated wave are distributed over the interval

$$
0 \leq \vartheta_{0} \leq \delta \vartheta_{0},
$$

where the initial phase size of the bunch is determined by the initial bunch length $l_{0}$ :

$$
\delta \vartheta_{0} \approx k l_{0}
$$

Here, $k=\frac{\omega_{0}}{c}=2 \pi / \lambda$. Thus, spontaneous emission is possible (the bunch is ready for radiation) when the initial bunch length is shorter than the wavelength. In terms of Fig. 3, the ready-for-radiation bunch means that the phase size of the bunch is small enough $\left(\delta \vartheta_{0}<2 \pi\right)$, so that most of the electrons in the bunch are in the decelerating phase of the radiated wave and, therefore, pass their kinetic energy to the wave. For instance, a dense electron bunch with a duration of $l_{0} / c \sim 1 \mathrm{ps}$ (with the initial bunch length of $l_{0} \sim 0.3 \mathrm{~mm}$ ) can be used for spontaneous coherent emission of a wave at a frequency of approximately $1 \mathrm{THz}$ or lower.

A key problem in dense bunches is a strong Coulomb repulsion, which leads to the increase of the axial bunch length. If the "operating" radiation mechanism of the subTHz source is based on longitudinal electron bunching [15] (either ubitron or Cherenkov radiation), then axial expansion of the bunch leads automatically to an increase in the bunch phase size with respect to the radiated wave [Fig. 3(a)].

In the case of the cyclotron maser, the Coulomb repulsion also provides an increase in the length of the bunch. However, the situation is more complicated due to the 2D (azimuthal-longitudinal) character of the electron bunching in the field of the wave. In this case, an effect of phase compensation of the Coulomb expansion can be provided. This effect is illustrated in Fig. 3(b). In contrast to ubitron and Cherenkov masers, where the places of equal phases of different electrons with respect to the wave represent a set of points $z_{n} \approx n \lambda$ [Fig. 2(a)], in the case of the cyclotron radiation the points of equal electron phases with respect to the wave $\vartheta=$ const form a 2D curve [Fig. 2(b)]. According to Eq. (2), this curve is a rotating helix $h_{0} z+\varphi=$ const. We show that it is possible to provide the situation when, as a result of Coulomb repulsion, the electron bunch spreads along the helix of the constant phase. In this case, an increase in the bunch length does not lead to an increase of the phase size of the bunch.

Let us consider evolution of the bunch in the case when the radiation fields are negligibly small, such that electrons 
(a)
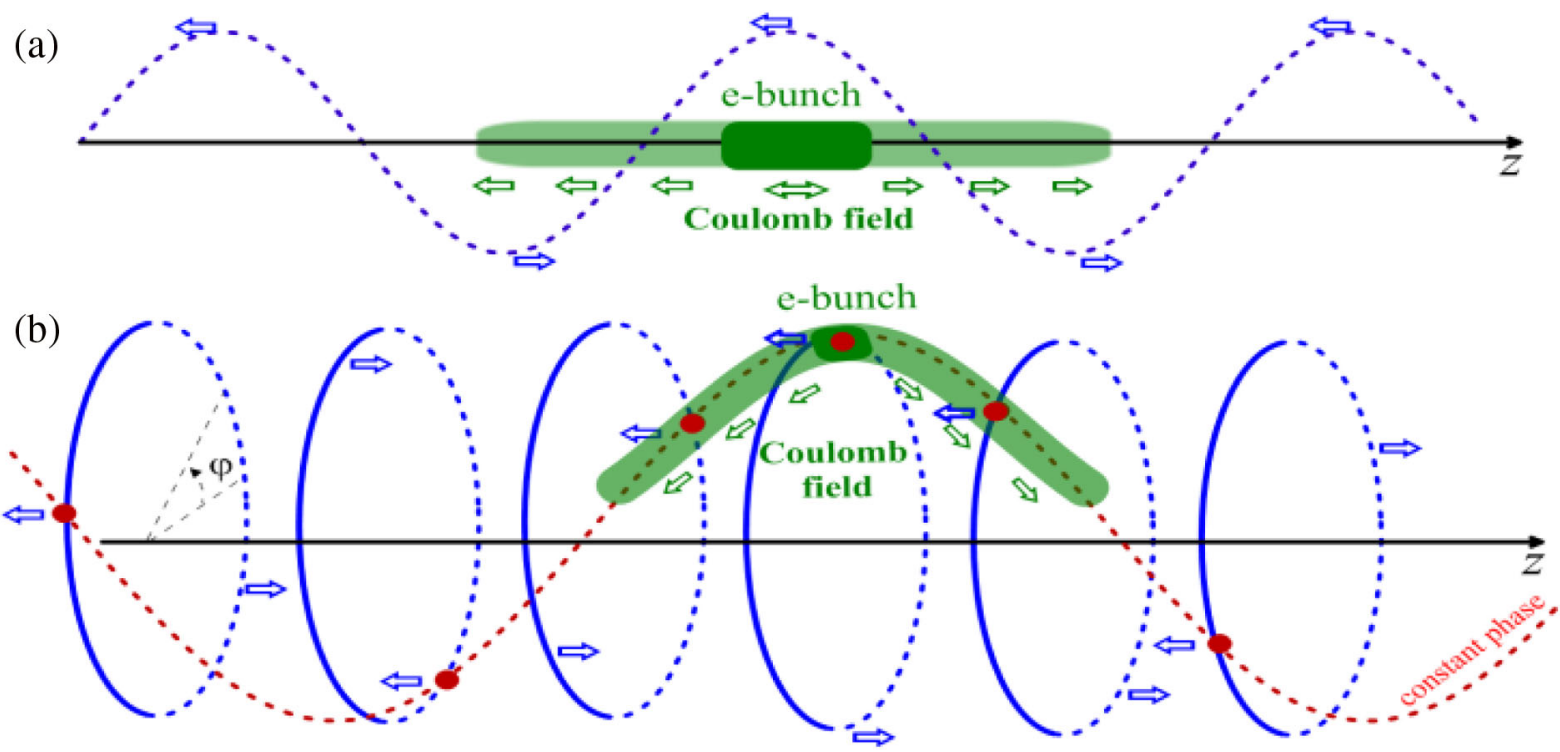

FIG. 3. (a) In the case of either ubitron or Cherenkov masers, axial expansion of the bunch leads to an increase in the bunch phase size with respect to the radiated wave. (b) In the cyclotron maser, the bunch is stretched along the 2D helix of the constant electron phase with respect to the wave.

move mainly only under the influence of the Coulomb field existing inside the dense bunch. Our aim is to find the dependence of the phase size of the bunch, $\delta \vartheta$, on the normalized axial length $k l$. According to Eq. (3), the initial values of these two sizes are the same. However, the dynamics of their changes under the effect of the Coulomb field of the bunch are different.

Let us consider electrons placed in the front and in the tail of the bunch (particles 1 and 2 in Fig. 4, respectively). Since particle 1 is accelerated by the Coulomb field and particle 2 is decelerated, the axial length of the bunch, $l=z_{1}-z_{2}$, increases in time due to the Coulomb repulsion. Having considered the axial electron motion in the ultrarelativistic approximation,

$$
\frac{d(k z)}{d\left(\omega_{0} t\right)}=\beta_{\|} \approx \beta_{\| 0}+\frac{\gamma-\gamma_{0}}{\beta_{\| 0} \gamma_{0}^{3}}
$$

one obtains the following equations describing the evolution of the axial bunch length:

$$
\frac{d(k l)}{d\left(\omega_{0} t\right)} \approx \frac{\gamma_{1-} \gamma_{2}}{\beta_{\| 0} \gamma_{0}^{3}} .
$$

Here, $\beta_{\|}=V_{\|} / c$ is the normalized axial electron velocity and $\left(\gamma-\gamma_{0}\right)$ describes the change in electron energy caused by the Coulomb repulsion.

The evolution in time of the phase of the two particles given by Eq. (2) is described by the following equation:

$$
\frac{d \vartheta}{d\left(\omega_{0} t\right)}=1-\beta_{\mathrm{gr}} \beta_{\|}-\frac{\Omega_{c}}{\omega_{0} \gamma}
$$

Here, $\beta_{\mathrm{gr}}=c h_{0} / \omega_{0}=V_{\mathrm{gr}} / c$ is the normalized group velocity of the wave. In the right-hand part of Eq. (5), the
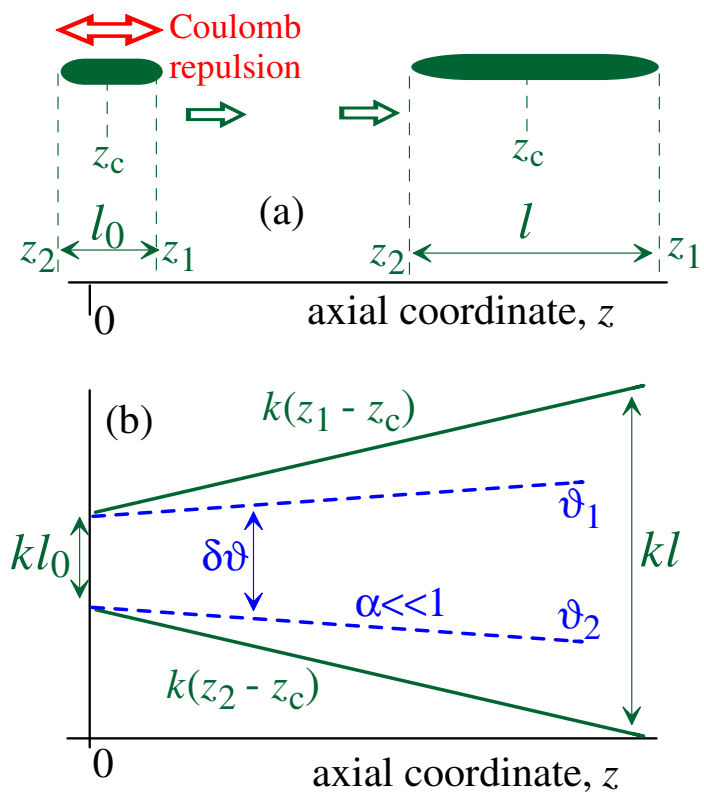

FIG. 4. (a) Enhancement of the axial length of the electron bunch in the process of its motion due to the Coulomb repulsion. (b) Characteristic evolution of axial coordinates of the electrons placed in the front and in the tail of the bunch (particles 1 and 2, respectively), as well as the evolution of phases of these two particles in the case of a small factor $\alpha$. Here, $z_{c}$ denotes the axial coordinate of the bunch center. 
term $\vartheta_{\|}^{\prime}=\beta_{\mathrm{gr}} \beta_{\|}$describes the change in the axial component of the electron cyclotron phase with respect to the wave $h_{0} z$, whereas the term $\vartheta_{\perp}^{\prime}=\frac{\Omega_{c}}{\omega_{0} \gamma}$ corresponds to the variation of the azimuthal component $\varphi$ in formula (1). Evidently, a change in the electron energy caused by the Coulomb repulsion results in opposite changes in $\vartheta_{\|}^{\prime}$ and $\vartheta_{\perp}^{\prime}$; namely, an increase (decrease) in the energy leads to an increase (decrease) in $\vartheta_{\|}^{\prime}$ but to a decrease (increase) in $\vartheta_{\perp}^{\prime}$.

This fact can be used to provide compensation of the effect of Coulomb repulsion on the electron cyclotron phase with respect to the wave. Let us find when this compensation is achieved. In this consideration, we neglect the effect of the radiated wave on the electron motion. We assume that the magnetic field corresponds to the exact cyclotron resonance:

$$
\frac{\Omega_{c, \text { res }}}{\gamma_{0} \omega_{0}}=1-\beta_{\mathrm{gr}} \beta_{\|, 0} .
$$

Then, the change in the resonant cyclotron phase caused by the Coulomb interaction is described as follows:

$$
\frac{d \vartheta}{d\left(\omega_{0} t\right)} \approx \mu\left(\gamma_{0}\right) \times\left(\gamma-\gamma_{0}\right)
$$

where

$$
\mu=-\frac{d}{d \gamma}\left(\beta_{\mathrm{gr}} \beta_{\|}+\frac{\Omega_{c}}{\omega_{0} \gamma}\right)
$$

We introduce axial and transverse components of the normalized relativistic electron momentum $p_{\|, \perp}=\gamma \beta_{\|, \perp}$ and transform Eq. (7) to the following form:

$$
\mu=-\frac{d}{d \gamma}\left(\beta_{\mathrm{gr}} \beta_{\|}+\frac{\Omega_{c}}{\omega_{0} \gamma}\right) .
$$

We assume that the Coulomb repulsion has basically axial character. In this situation, the general relativistic formula

$$
\gamma^{2}=1+p_{\|}^{2}+p_{\perp}^{2}
$$

leads to

$$
\frac{d p_{\|}}{d \gamma}=\gamma / p_{\|}=\frac{1}{\beta_{\|}} .
$$

Then, formula (8) together with Eqs. (6) and (9) leads to the following relation:

$$
\mu\left(\gamma_{0}\right)=\frac{1}{\gamma_{0}}\left(\frac{\beta_{\mathrm{gr}}}{\beta_{\|, 0}}-1\right) .
$$

This factor determines the evolution of the phase size of the bunch, $\delta \vartheta=\vartheta_{1}-\vartheta_{2}$, in time:

$$
\frac{d(\delta \vartheta)}{d\left(\omega_{0} t\right)} \approx \mu\left(\gamma_{0}\right) \times\left(\gamma_{1}-\gamma_{2}\right) .
$$

Having compared this equation and Eq. (4), one obtains the following relations between changes in the phase size and in the axial bunch length:

$$
\frac{d(\delta \vartheta)}{d(k l)} \approx \alpha=\frac{\gamma_{0}^{2}}{\beta_{\|, 0}}\left(\frac{\beta_{\mathrm{gr}}}{\beta_{\|, 0}}-1\right) .
$$

This formula is illustrated in Fig. 4. Though the initial values of the phase size and of the normalized axial length are the same, the rates of increase of these two sizes in time due to the Coulomb repulsion are different, namely,

$$
\delta \vartheta=k l_{0}+\alpha \times k\left(l-l_{0}\right) .
$$

In the case of the group resonance,

$$
\beta_{\mathrm{gr}}=\beta_{\|, 0},
$$

The factor $\alpha$ is small, so that the Coulomb repulsion leads to a very slow change of the phase size of the bunch. Let us emphasize that, naturally, in this situation the increase in the length of the electron bunch due to the Coulomb repulsion is not avoided. However, this Coulomb expansion does not lead to a significant increase in the phase size of the bunch [Fig. 4(b)], as electrons expand along the curve (helix) of the constant phase with respect to the radiated wave [Fig. 3(b)].

Note that, when we obtain this equation, we do not use any particular model describing the Coulomb field inside the electron bunch. In other words, it does not matter how the difference in the energies $\left(\gamma_{1}-\gamma_{2}\right)$ of the extreme particles of the bunch grows in time due to the Coulomb repulsion. Therefore, Eq. (10) describing the compensation of the effect of the Coulomb field on different (transverse and axial) components of the electron cyclotron phase has a very general character. Note also that the effect of the total compensation in the case of the group resonance is weakly sensitive to the spread in initial electron energy, $d \gamma_{0}$. Actually, if Eq. (11) is fulfilled for the central energy fraction, then the initial energy spread $d \gamma_{0}$ leads to the following spread in the parameter $\alpha$ :

$$
d \alpha \approx \frac{d \gamma_{0}}{\gamma_{0}} .
$$

For modern photoinjectors, this value is as small as approximately $1 \%$ [1]. 

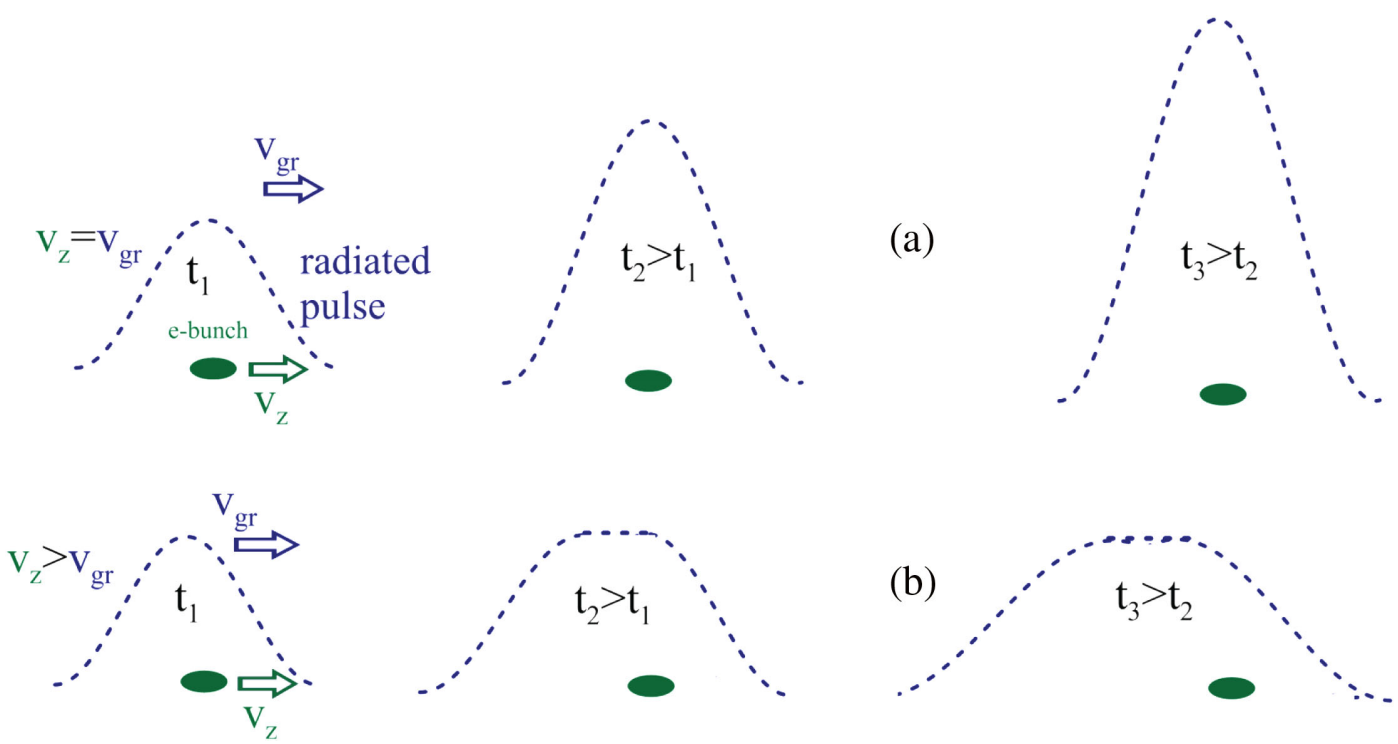

FIG. 5. (a) The radiated wave pulse in the case of the superradiant emission in the group resonance regime. (b) The radiated pulse "slips" with respect to the electron bunch in the case when the electron velocity differs from the wave group velocity.

A principal point is that the group resonance regime described by condition (11) is optimal also from the viewpoint of organizing the wave emission process. In this regime, quanta emitted in different moments of time do not run away from the electron bunch, which leads to the accumulation of radiation in the region close to the bunch and, as a result, to the formation of a powerful short wave pulse propagating together with the bunch (Fig. 5). A rapid increase of the wave amplitude in the region of the short electron bunch provides the maximal growth rate of the cyclotron instability [21]. This is analogous to the superradiant radiation effects, studied both theoretically and experimentally for various types of electron masers $[25,26]$ operating in the traditional regime of the induced emission from relatively long electron bunches. In the case of the spontaneous emission from a short bunch studied in this paper, this advantage of the superradiant regime is naturally combined with the solution of the problem of the axial expansion of the bunch due to the Coulomb repulsion.

We would like to note here the analogy between the above effect of compensation of the influence of Coulomb forces on the phase of a particle relative to the radiated wave and the autoresonance effect known in the theory of electron cyclotron masers and that is the basis of the socalled cyclotron autoresonance masers [22-24]. The latter represents the compensation of the influence of the field of the radiated wave on the electron phase. It can be described by the same formalism as above, but with one amendment. Namely, one should change Eq. (9) that relates changes in the energy of a particle and its longitudinal momentum, taking into account that in the case of the electron motion in the field of the radiated wave

$$
\frac{d p_{\|}}{d \gamma}=\frac{1}{\beta_{\mathrm{ph}}}=\beta_{\mathrm{gr}} .
$$

Here, $\beta_{\mathrm{ph}}=\omega_{0} / c h_{0}=V_{\mathrm{ph}} / c$ is the normalized phase velocity of the wave. Correspondingly, one should substitute $\frac{1}{\beta_{\|, 0}}$ with $\beta_{\mathrm{gr}}$ in Eqs. (10) and (11), so that $\alpha \sim\left(\beta_{\mathrm{gr}}^{2}-1\right)$ and the autoresonance effect takes place when the group velocity of the wave coincides with the speed of light, $\beta_{\mathrm{gr}}=1$ (more precisely and more generally, this condition should be satisfied for the phase velocity, $\beta_{\mathrm{ph}}=1$ ).

The effect described in this paper has the same nature, namely, the mutual compensation of changes in the longitudinal and transverse components of the electron phases relative to the radiated wave. It turns out, however, that if these changes are caused not by the fields of the radiated wave but by quasistatic Coulomb fields of repulsion in a dense short electron bunch, then the compensation effect occurs if the group velocity of the wave coincides not with the speed of light but with the speed of translational motion of electrons.

\section{EQUATIONS OF ELECTRON MOTION IN RADIATED AND COULOMB FIELDS}

We start from the equation for the change in the electron relativistic gamma factor,

$$
m c^{2} \frac{d \gamma}{d t}=-e(\boldsymbol{V} \cdot \boldsymbol{E})
$$

where $\boldsymbol{V}$ is the electron velocity and $\boldsymbol{E}$ is the total electric field acting on the particle. Let us represent the right-hand part of this equation as a sum of two terms: 


$$
\frac{d \gamma}{d\left(\omega_{0} t\right)}=-F_{c}-F_{w}
$$

Here,

$$
F_{c}=\frac{e}{\omega_{0} m c^{2}} E_{\|, c} V_{\|} \quad \text { and } \quad F_{w}=\frac{e}{\omega_{0} m c^{2}} \boldsymbol{E}_{\perp, w} \cdot \boldsymbol{V}_{\perp}
$$

describe the interaction of the particle with the axial Coulomb electric field $E_{\|, c}$ and with the transverse electric field $E_{\perp, w}$ of the radiated wave, respectively. From the very beginning, we normalize the time to the base frequency of the radiated wave packet $\omega_{0}$.

\section{A. Coulomb interaction}

In our model, we take into account only the axial interaction of electrons with the Coulomb field. This restriction is due to the relativistic compensation of contributions from electron and magnetic fields in the transverse component of the Lorentz force, as well as due to the quasi1D character of the electron bunch. The latter is true in the situation when the transverse component of the electron velocity $V_{\perp}$ is small as compared to the axial component $V_{\|}$, and when the bunch radius is small enough as compared to the bunch length in the copropagated reference system. In this approximation, the axial Coulomb electric field inside the bunch can be easily calculated by using the bunch model described in detail in Ref. [18].

We represent the bunch as a discrete set of $n$ fractions (thin "pancakes", Fig. 6). Each pancake has the electric charge $q / n$, where $q$ is the total charge of the bunch. In the reference system related with the electron bunch, the axial electric field of the $j$ th pancake at the axis of the symmetry is determined by the following formula:

$$
E_{\|, j}^{\prime}=\frac{2 \pi q}{n S}\left(1-\frac{1}{\sqrt{\frac{R^{2}}{\Delta z_{i, j}^{\prime}}+1}}\right) .
$$
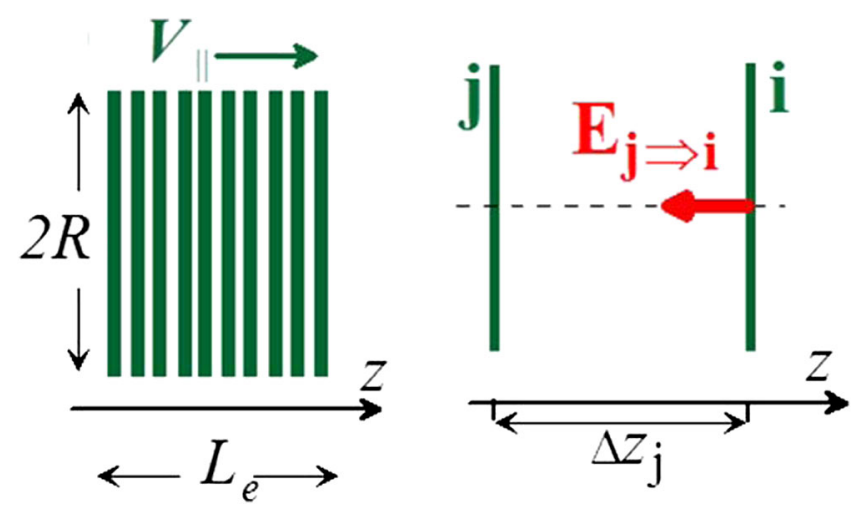

FIG. 6. Calculation of the Coulomb field in the electron bunch: the model of a set of charged pancakes.
Here, $S=\pi R^{2}, R$ is the radius of the electron bunch, $\Delta z_{i, j}^{\prime}=z_{i}^{\prime}-z_{j}^{\prime}$, and $z_{i}^{\prime}$ and $z_{j}^{\prime}$ are the coordinates of the $i$ th and $j$ th pancakes, respectively, in the correlated system. The distance between two disks in a correlated system is connected with the same in the laboratory coordinate system by the Lorentz relation $\Delta z_{i, j}^{\prime}=\gamma_{\|, i} \Delta z_{i, j}$, where $\Delta z_{i, j}$ is the distance between the $i$ th and $j$ th disks in the laboratory coordinate system and $\gamma_{\|}=1 / \sqrt{1-\beta_{\|}^{2}}$ is the longitudinal Lorentz factor of the $i$ th pancake. The total electric field acting on the $j$ th pancake is found as a sum over all pancakes. Therefore, the Coulomb field contribution in Eq. (12), $F_{c, j}$, for an electron placed in the $j$ th pancake is determined by the formula

$$
F_{c, j}=\chi_{c, j} \Xi_{j}
$$

Here

$$
\begin{aligned}
\chi_{c, j} & =\frac{I L_{e, 0} \lambda \beta_{\|, j}}{I_{a} S}, \\
\Xi_{j} & =\sum_{i \neq j}^{n} \operatorname{sgn}\left(z_{j}-z_{i}\right)\left(1-\frac{\left|\Delta z_{i, j} \gamma_{\|, i}\right|}{\sqrt{R^{2}+\left(\Delta z_{i, j} \gamma_{\|, i}\right)^{2}}}\right) f_{q}\left(z_{i, 0}\right),
\end{aligned}
$$

where $I=q c / L_{e, 0}$ is the electron current, $L_{e, 0}$ is the initial length of the bunch, $\lambda$ is the wavelength, $I_{a}=m c^{3} / e$, and $f_{q}\left(z_{i, 0}\right)$ is the function of the charge distribution in the bunch (in simulations, the Gaussian distribution function is used).

For the normalized electron axial momentum $p_{\|}=\gamma \beta_{\|}$, coming from the results for the Lorentz-factor changes (14), taking into account the relation between the axial moment and energy, caused by Coulomb interaction,

$$
\frac{d p_{\|, j}}{d\left(\omega_{0} t\right)}=-\frac{F_{c, j}}{\beta_{\|, j}}
$$

\section{B. Electron-wave interaction}

In order to describe the electron-wave interaction, we use a traditional approach of the cyclotron resonance maser theory [22]. We consider the motion of a particle along a helical trajectory in a circular waveguide immersed in the uniform magnetic field $\boldsymbol{B}_{0}$ (Fig. 2). If the particle rotates around the waveguide axis, then the complex transverse electron coordinate and the complex transverse of the velocity are described as follows:

$$
\begin{aligned}
& r_{+}=x+i y=r_{c} e^{i \varphi}, \\
& V_{+}=V_{x}+i V_{y}=i V_{\perp} e^{i \varphi}, \quad \varphi=\int \Omega_{\mathrm{c}} / \gamma d t .
\end{aligned}
$$

Here, $(\varphi, r, z)$ are the cylindrical coordinates, $r_{c}$ is the Larmor radius, and $V_{\perp}=r_{c} \Omega_{\mathrm{c}}$ is the oscillatory velocity. 
We assume that the particle interacts with the lowest $\mathrm{TE}_{11}$ circular-polarized transverse mode of the waveguide. The field of this wave is described by the following vector potential:

$\boldsymbol{A}=\operatorname{Re}\left(k_{\perp} \frac{m_{e} c^{2}}{e} J_{1}\left(k_{\perp} r\right) a(t, z) \exp (i \omega t-i h z)\right)$,

where $a(t, z)$ is the normalized (slow) amplitude of the wave; $k_{\perp}=\sqrt{k_{0}^{2}-h_{0}^{2}}=\mu_{11} / R_{w}$ is the transverse wave number, $k_{0}$ is the free-space wave number, $\mu_{11}$ is the root of the equation $J_{1}^{\prime}\left(\mu_{11}\right)=0$, and $R_{w}$ is the waveguide radius.

Consequently, transverse complex electric and magnetic wave fields $E_{+}=E_{x}+i E_{y}, B_{+}=B_{x}+i B_{y}$ are determined as follows:

$$
\begin{aligned}
E_{+}= & -i * k_{\perp} \frac{m_{e} c^{2}}{e} * \exp (i \varphi)\left[\frac{\partial}{\partial r}+\frac{i}{r} \frac{\partial}{\partial \varphi}\right] \\
& \times \operatorname{Re}[a(t, z) \exp (i \omega t-i h z)] J_{1}\left(k_{\perp} r\right), \\
B_{+}= & i h_{0} / k_{0} E_{+},
\end{aligned}
$$

and in the center of the waveguide (the bunch place) transverse complex electric and magnetic wave fields are fields of a plane wave:

$$
E_{+}=-\frac{i}{2} \frac{m c^{2}}{e} a(t, z) \exp (i \varphi-i \omega t+i h z) .
$$

The equation for the electron energy change,

$$
m c^{2} \frac{d \gamma}{d t}=-e \operatorname{Re}\left(E_{+}^{*} V_{+}\right),
$$

leads to the following equation:

$$
\frac{d \gamma}{d\left(\omega_{0} t\right)}=-F_{w}
$$

Here, $F_{w}=\frac{e}{\omega_{0} m c^{2}} \operatorname{Re}\left(E_{+}^{*} V_{+}\right)=\operatorname{Re}\left[\chi_{w} a \exp (i \vartheta)\right], \chi_{w}=$ $\beta_{\perp} / 2$ is the electron-wave coupling factor, and $\beta_{\perp}=V_{\perp} / c$ is the normalized transverse velocity.

Electron-wave interaction leads to a change in the normalized axial momentum $p_{\|}=\gamma \beta_{\|}$:

$$
\frac{d p_{\|}}{d t}=\frac{e}{m c^{2}} \operatorname{Im}\left(B_{+}^{*} V_{+}\right) \text {. }
$$

Having compared this equation and Eq. (19) and taking into account the ratio between wave electric and magnetic fields in Eq. (18), one obtains

$$
\frac{d p_{\|}}{d\left(\omega_{0} t\right)}=-\beta_{\mathrm{gr}} F_{w}
$$

\section{Equations of the electron motion}

The equation for the total electron energy change (12) is transformed by relations (14) and (19) to the following equation:

$$
\frac{d \gamma_{j}}{d\left(\omega_{0} t\right)}=-\operatorname{Re}\left[\chi_{w j} a \times \exp (i \vartheta)\right]-F_{c, j}
$$

For the normalized electron axial momentum in the $j$ th disk, coming from the results (15) and (20), taking into account the ratio between wave electric and magnetic fields (17):

$$
\frac{d p_{\|, j}}{d\left(\omega_{0} t\right)}=-\beta_{\mathrm{gr}} \operatorname{Re}\left[\chi_{w, j} a \times \exp (i \vartheta)\right]-\frac{F_{c, j}}{\beta_{\|, j}} .
$$

The change in the complex transverse momentum, $p_{+}=p_{\perp} e^{i \varphi}$, is determined by the following equation:

$$
\frac{d p_{+}}{d\left(\omega_{0} t\right)}=-\frac{1}{\omega_{0}} \times \frac{e}{m c^{2}}\left(E_{+}+\beta_{\|} B_{+}+\frac{p_{+}}{\gamma} B_{0}\right) .
$$

The equation for the electron cyclotron phase change is

$$
\begin{aligned}
\frac{d \varphi}{d\left(\omega_{0} t\right)}= & -\frac{1}{\omega_{0} p_{\perp}} \times \frac{e}{m c^{2}} \\
& \times \operatorname{Im}\left(E_{+} \exp (i \varphi)+\beta_{\|, 0} B_{+} \exp (i \varphi)-\frac{p_{\perp}}{\gamma} B_{0}\right) \\
= & \frac{b}{\gamma}-\left(1-\beta_{\|, 0} \beta_{\mathrm{gr}}\right) \operatorname{Im}\left(\frac{a}{p_{\perp}} \exp (i \vartheta)\right) .
\end{aligned}
$$

Here, $b=\Omega_{c} / \omega_{0}$. The electron resonance phase is the difference of the electron phase with respect to the wave and the electron cyclotron phase: $\vartheta=\omega t-h z-\varphi$. The equation for the change in the resonance phase is

$$
\begin{aligned}
\frac{d \vartheta}{d\left(\omega_{0} t\right)}= & -\beta_{\mathrm{gr}}\left(\beta_{\|}-\beta_{\|, 0}\right)-b\left(\frac{1}{\gamma}-\frac{1}{\gamma_{0}}\right) \\
& +\left(1-\beta_{\|, 0} \beta_{\mathrm{gr}}\right) \operatorname{Im}\left(\frac{a}{p_{\perp}} \exp (i \vartheta)\right)+\Delta .
\end{aligned}
$$

Here, $\Delta=1-\beta_{\mathrm{gr}} \beta_{\|, 0}-b / \gamma_{0}$ is the mismatch of the electron-cyclotron resonance.

\section{Equation of the superradiant radiation of the resonant wave}

In order to describe the spatiotemporal evolution of the slow rf wave amplitude $a(z, t)$, we follow the way described in detail in Ref. [27]. We consider the wave equation for the vector potential: 


$$
\frac{1}{c^{2}} \frac{\partial^{2} \boldsymbol{A}}{\partial t^{2}}-\frac{\partial^{2} \boldsymbol{A}}{\partial z^{2}}-\Delta_{\perp} \boldsymbol{A}=\frac{4 \pi}{c} \boldsymbol{J}
$$

This equation leads to the following equation for the slow rf wave amplitude:

$$
\frac{1}{c^{2}} \frac{\partial^{2} a}{\partial t^{2}}+\frac{2 i \omega_{0}}{c^{2}} \frac{\partial a}{\partial t}-\frac{\partial^{2} a}{\partial z^{2}}+2 i h_{0} \frac{\partial a}{\partial z}=i g \rho_{n} Z(z),
$$

where $g=2 I\left(1-\beta_{\mathrm{gr}}{ }^{2}\right) / I_{a} N \beta_{\mathrm{gr}}$ is the excitation factor, $N=$ 0.4 is the norm of the operating $\mathrm{TE}_{11}$ transverse mode, and the function $Z(z)$ describes the bunch axial position, namely, $Z(z)=1$ if $z-V_{\|} t \in\left[0, L_{e}\right]$ and $Z(z)=0$ if $z-V_{\|} t \notin\left[0, L_{e}\right]$. The source in Eq. (26) is proportional to the factor of the electron bunching with respect to the radiated wave:

$$
\rho_{n}=\left\langle\chi_{w} \exp (-i \vartheta)\right\rangle
$$

where $\langle\cdots\rangle$ denotes averaging over the whole electron ensemble. This equation represents the first harmonic of the Fourier transformation of the electron current density over the cyclotron phases of electrons with respect to the operating wave and is analogous to the form factor of a short bunch in the FEL theory.

If we use the following normalized variables:

$$
\zeta=k_{0}\left(z-V_{\|, 0} t\right), \quad \tau=\omega_{0}\left(t-V_{\|, 0} z / c^{2}\right),
$$

then $\frac{d}{d\left(\omega_{0} t\right)} \rightarrow \frac{1}{\gamma_{\|, 0}} \frac{\partial}{\partial \tau}$, and the wave equation (26) is transformed as follows:

$$
\frac{\partial^{2} a}{\partial \tau^{2}}-\frac{\partial^{2} a}{\partial \zeta^{2}}+2 i s \frac{\partial a}{\partial \tau}-2 i \varepsilon \frac{\partial a}{\partial \zeta}=i G \rho_{n} \frac{Z(\zeta)}{L_{e}}
$$

Here, $s=\left(1-\beta_{\mathrm{gr}} \beta_{\|}\right) /\left(1-\beta_{\|}^{2}\right)$ is a factor, which is close to unit in the case when $\beta_{\mathrm{gr}} \approx \beta_{\|}, \varepsilon \approx\left(\beta_{\|}-\beta_{\mathrm{gr}}\right) \gamma_{\|, 0}{ }^{2}$ is the factor describing "slippage" of the radiated wave pulse with respect to electrons, and $G=$ $2 k_{0} L_{e} I\left(1-\beta_{\mathrm{gr}}{ }^{2}\right) \gamma_{\|, 0}^{2} / I_{a} N \beta_{\mathrm{gr}}$ is the factor of the wave excitation.

We consider emission from a very short electron bunch, $L_{e} \ll \lambda \gamma_{\|, 0}{ }^{2}$. In this case, we can use the approximation $Z(\zeta) \approx L_{e} \delta(\zeta)$, so that Eq. (28) is reduced as follows:

$$
2 i \frac{\partial a}{\partial \tau}-2 i \varepsilon \frac{\partial a}{\partial \zeta}-\frac{\partial^{2} a}{\partial \zeta^{2}}=i G \rho_{n} \delta(\zeta)
$$

At the zero initial conditions, this equation can be solved analytically $[27,28]$ :

$$
\begin{aligned}
a= & \frac{G}{2} \sqrt{\frac{i}{\pi}} \int_{0}^{\tau} \frac{\rho_{n}}{\sqrt{\tau-\tau^{\prime}}} \\
& \times \exp \left[-\sqrt{2 i} \zeta^{2} /\left(\tau-\tau^{\prime}\right)-i \varepsilon^{2}\left(\tau-\tau^{\prime}\right) / 2-i \varepsilon \zeta\right] d \tau^{\prime} .
\end{aligned}
$$

In the case of the exact group resonance regime, $\beta_{\mathrm{gr}}=\beta_{\|}$, the rf-wave amplitude in the location of the bunch $\zeta=0$ is found as follows:

$$
a(\zeta=0, \tau)=\frac{G}{2} \sqrt{\frac{i}{\pi}} \int_{0}^{\tau} \frac{\rho_{n}}{\sqrt{\tau-\tau^{\prime}}} d \tau^{\prime} .
$$

Note that in this case the wave excitation factor is described by the following simplified formula:

$$
G=\frac{2 k_{0} L_{e} I}{I_{a} N \beta_{\mathrm{gr}}} .
$$

\section{SIMULATIONS OF THE CYCLOTRON SUPERRADIANT RADIATION FROM SHORT DENSE BUNCHES}

In simulations, we describe electron motion by the equation for the electron energy (21), the equation for the electron axial momentum (22), and the equation for the electron cyclotron phase with respect to the wave (25). In these equations, the rf wave amplitude $a$ is determined by Eq. (31), and this is the same for all electrons of the short bunch. As for the normalized Coulomb field acting on the $j$ th particle, $F_{c, j}$, this is determined by Eq. (14). Naturally, this model includes a number of approximations; namely, a very simple pancake model is used for description of the Coulomb field, a pointlike electron bunch model is used to describe the wave excitation by means of Eq. (31), and no initial energy spread is taken into account. However, such a simple approach can give predictions which are in very good agreement with result simulations on the basis of a "full" particle-in-cell code based on the "direct" modeling of Maxwell equations [17]. As for the initial energy spread, it is mentioned above that a spread of $1 \%$ typical for modern photoinjectors is not too important for effects studied in this paper. More importantly, such a spread is significantly smaller than the dynamic spread that occurs quickly enough in the short dense bunch due to the Coulomb repulsion.

We consider electron bunches with the total charge of $0.1-1.0 \mathrm{nC}, 1 \mathrm{~mm}$ diameter, and the electron energy is $6 \mathrm{MeV}$ (which corresponds to the relativistic Lorentz factor of electrons $\gamma=13$ ). These parameters are quite typical for modern photoinjectors [1]; in particular, they are close to the expected parameters of the photoinjector the Israeli $\mathrm{THz}$ radiation source being constructed at Ariel University [29]. 
The initial transverse velocity $\beta_{\perp 0}=V_{\perp, 0} / c \approx 1 / \gamma_{0}$ is chosen from the viewpoint of maximization of the electron efficiency predicted by the cyclotron maser theory [22]. This transverse velocity together with the group resonance condition (11) results in the following formula describing the Doppler up-conversion of the nonrelativistic cyclotron frequency:

$$
\omega_{0} \approx \frac{\gamma \Omega_{c}}{2}
$$

for the cyclotron radiation at a wave frequency of $0.5 \mathrm{THz}$, which corresponds to the operating magnetic field $B_{0} \approx 2.7 \mathrm{~T}$.

Figure 7 illustrates simulations of spontaneous emission from short (the initial length is $L_{e, 0}=\lambda / 4$ ) bunches with
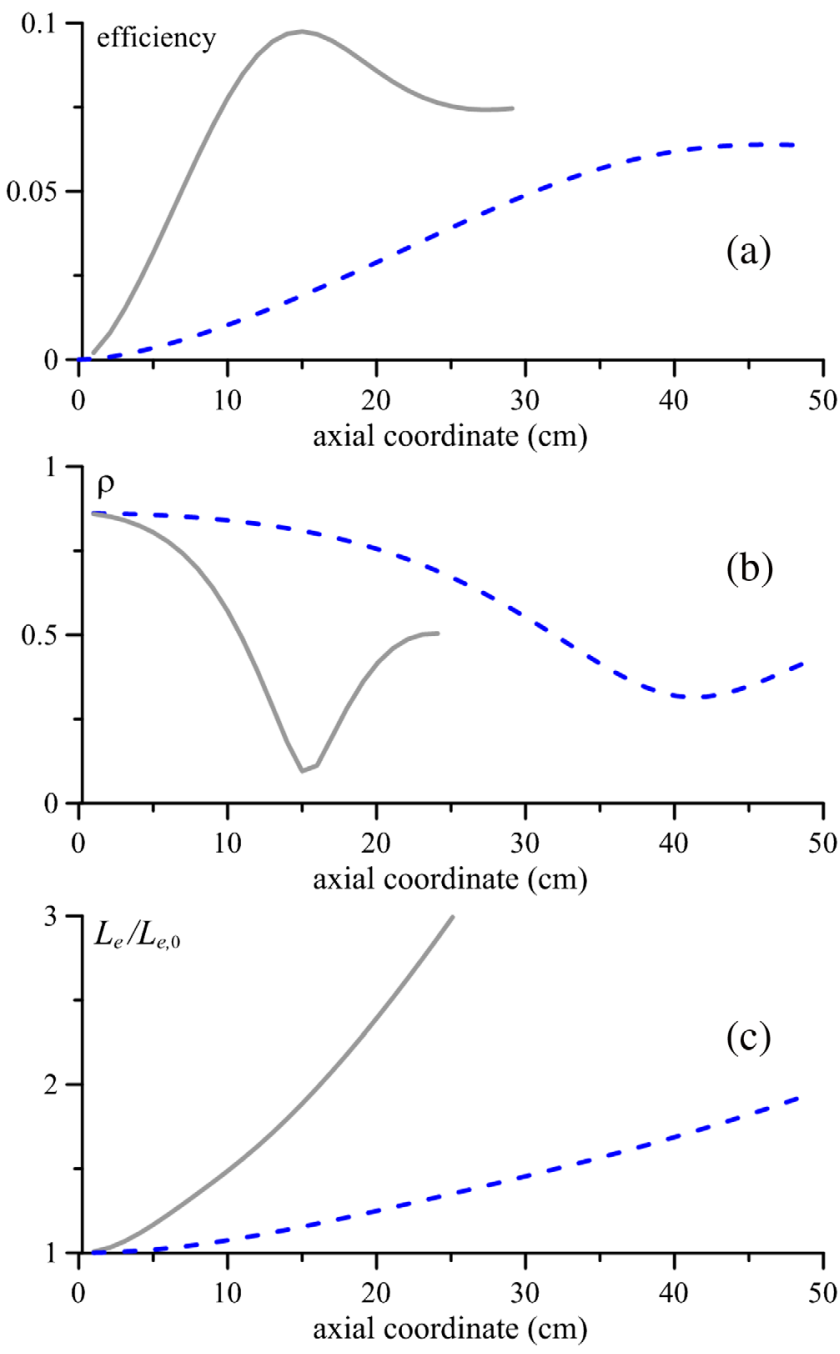

FIG. 7. Spontaneous emission from short $\left(L_{e, 0}=\lambda / 4\right)$ bunches with two different bunch charges 0.1 (solid curves) and $1 \mathrm{nC}$ (dashed curves). Efficiency of the electron-wave interaction (a), electron bunching efficiency $\rho$ (b), and the effective bunch axial length normalized to the initial length $L_{e} / L_{e, 0}$ (c) versus the axial coordinate. different charges $(0.1$ and $1.0 \mathrm{nC})$. It shows the electron efficiency [the change in electron energy averaged over all particles in the bunch normalized to the initial kinetic electron energy, $\left.\eta=\left\langle\gamma_{0}-\gamma\right\rangle /\left(\gamma_{0}-1\right)\right]$, the efficiency of electron bunching (which is described by formula $\rho=\left\langle e^{-i \vartheta}\right\rangle$ ), and the relative increase in the bunch length $L_{e} / L_{e, 0}$. In these simulations, the waveguide radius is chosen to provide the exact group resonance, so that the group velocity of the radiated wave coincides with the initial value of the axial component of the electron velocity.

The initial bunch length $L_{e, 0}=\lambda / 4$ corresponds to a relatively small initial phase size of the bunch $\delta \vartheta=\pi / 4$. This size corresponds to a quite high efficiency of the electron bunching at the input of the radiation region, $\rho(0) \sim 1$. In the process of motion of the bunch along the operating waveguide, the bunch length increases basically due to the Coulomb field. Even in the case of a relatively small charge $(0.1 \mathrm{nC})$, the bunch length increases by a factor of approximately 5 in the process of the motion of the bunch through the $50 \mathrm{~cm}$ radiation region [Fig. 7(c)]. However, as is predicted in Sec. II, such a dramatic increase in the bunch length does lead to a significant increase in the bunch phase size, so that the bunching efficiency remains at a level $\rho(z) \sim 0.5$ throughout the whole electron-wave interaction region. Since the wave amplification is determined by the stability of the bunch phase size $[\partial a / \partial \tau \propto \rho$; see Eq. (31)], this results in a stable increase in the electron efficiency with the axial coordinate of the moving bunch [Fig. 5(a)]. According to Eq. (31), while the electron bunching factor $\rho(z)$ remains approximately constant, the superradiative radiation process leads to the growth of the wave amplitude in time as $a(\tau) \propto \sqrt{\tau}$. In accordance with Eq. (21), this growth leads to the fast growth of the electron efficiency

$$
\eta(\tau) \propto \tau^{3 / 2}
$$

The saturation is achieved at relatively short lengths $(50 \mathrm{~cm}$ at $0.1 \mathrm{nC}$ and $15 \mathrm{~cm}$ at $1 \mathrm{nC}$ ), and the saturated values of the electron efficiency are relatively high (7\%-10\%) almost independently of the bunch charge.

In contrast to Figs. 7 and 8 illustrates the situation when the initial bunch length is close to the wavelength, length $L_{e, 0} \approx \lambda$. This corresponds to the initial phase size of the bunch $\delta \vartheta \approx 2 \pi$ and, correspondingly, to a close-tozero initial bunching factor $\rho(0)$. In fact, this case illustrates the transition from the spontaneous radiation of a short-length electron bunch to the induced radiation of an electron ensemble which is not prebunched with respect to the radiated wave. In this case of induced radiation, the bunching efficiency $\rho(z)$ increases with the axial coordinate due to bunching of electrons in the field of the radiated wave [Fig. 8(b)]. Similar to the previous 

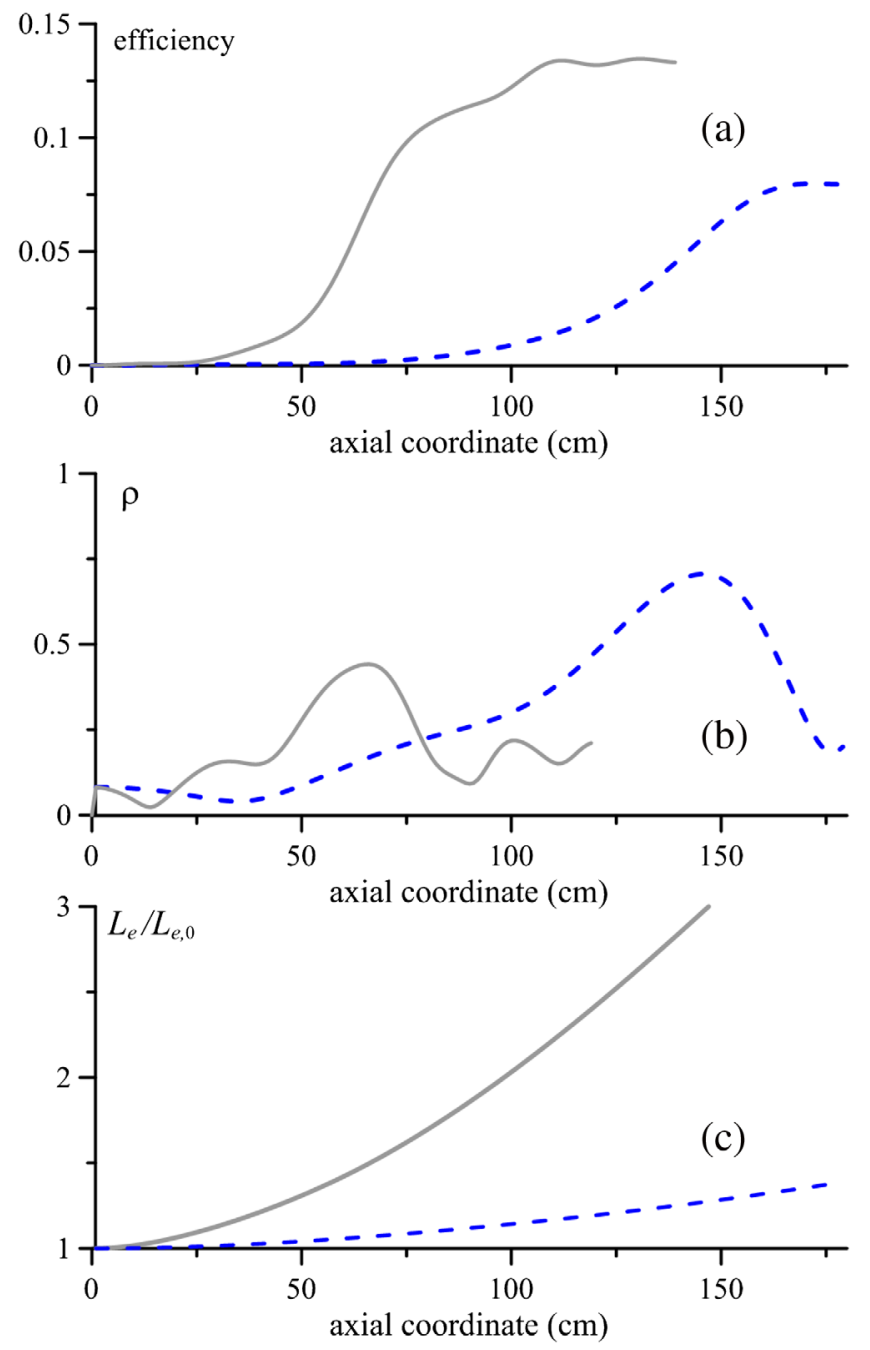

FIG. 8. Induced emission from relatively long $\left(L_{e, 0}=\lambda\right)$ bunches with two different bunch charges 0.1 (solid curves) and $1 \mathrm{nC}$ (dashed curves). Efficiency of the electron-wave interaction (a), electron bunching efficiency $\rho$ (b), and the effective bunch axial length normalized to the initial length $L_{e} / L_{e, 0}$ (c) versus the axial coordinate.

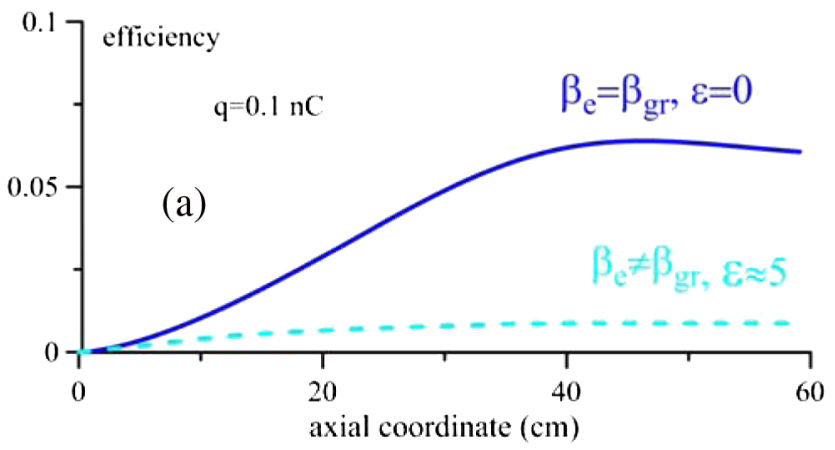

case, the bunch length increases due to the Coulomb repulsion [Fig. 8(c)]. However, due to the effect of the compensation of the Coulomb repulsion in the phase space, this repulsion does not prevent the phase bunching of the electron ensemble [Fig. 8(b)]. Because of this fact, the saturated efficiency in this case of induced radiation [Fig. 8(a)] is close to that in the previous case of spontaneous radiation [Fig. 7(a)]. However, in contrast to the fast growth of the electron efficiency in the case of spontaneous emission described by Eq. (32), the induced process provides a slow exponential growth of the efficiency [Fig. 8(a)]. This growth is very similar to the small-signal growth of the instability in FELs. Therefore, the saturation length of the induced process is significantly longer, which is naturally due to the need for additional time for the electron bunching process.

The group resonance regime is optimal from the viewpoint of radiation. The results of numerical simulation show (see Fig. 9) that a wave "slippage" leads to a significant decrease in efficiency: For the bunch charge $0.1 \mathrm{nC}$, efficiency decreases by a factor of approximately 6 [Fig. 9(a)]; for $1 \mathrm{nC}$, the efficiency decrease is slightly smaller than in the case of $0.1 \mathrm{nC}$ [see Fig. 9(b)]. Thus, in the case of a group resonance regime, we have an opportunity to produce rf pulses with the megawatt level of power and a duration of approximately 100 ps by using spontaneous coherent radiation (see Fig. 10).

We have marked above that the efficiency of spontaneous radiation is close to the induced radiation efficiency, but the saturation length of the induced process is significantly longer and depends on the initial signal formed by bunch edges. The additional advantage of spontaneous radiation is revealed in the cases of sufficiently great bunch charges. The results of numerical simulations shown in Fig. 11 illustrate that a bunch charge increase leads to a decrease in the induced radiation efficiency and to the constant efficiency of the spontaneous radiation.

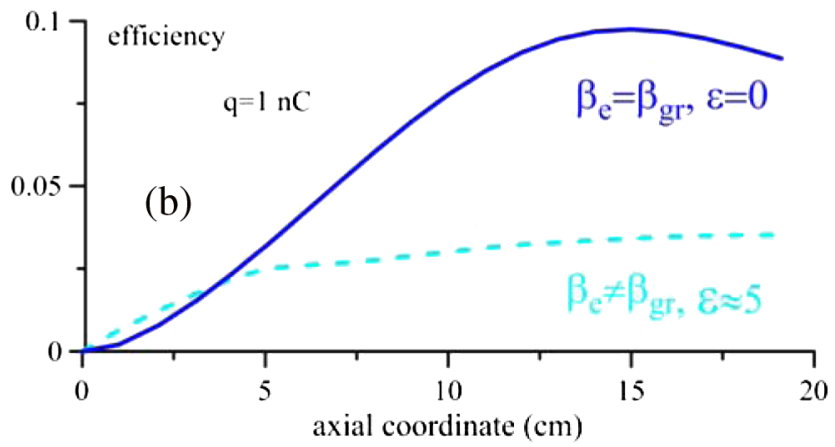

FIG. 9. Spontaneous emission from short $\left(L_{e, 0}=\lambda / 4\right)$ bunches with two different bunch charges 0.1 (a) and $1 \mathrm{nC}$ (b). Efficiency of the electron-wave interaction versus the axial coordinate. Solid curves describe the regime of the exact group resonance, and dashed curves illustrate a case when the group wave velocity is smaller than the bunch velocity. 

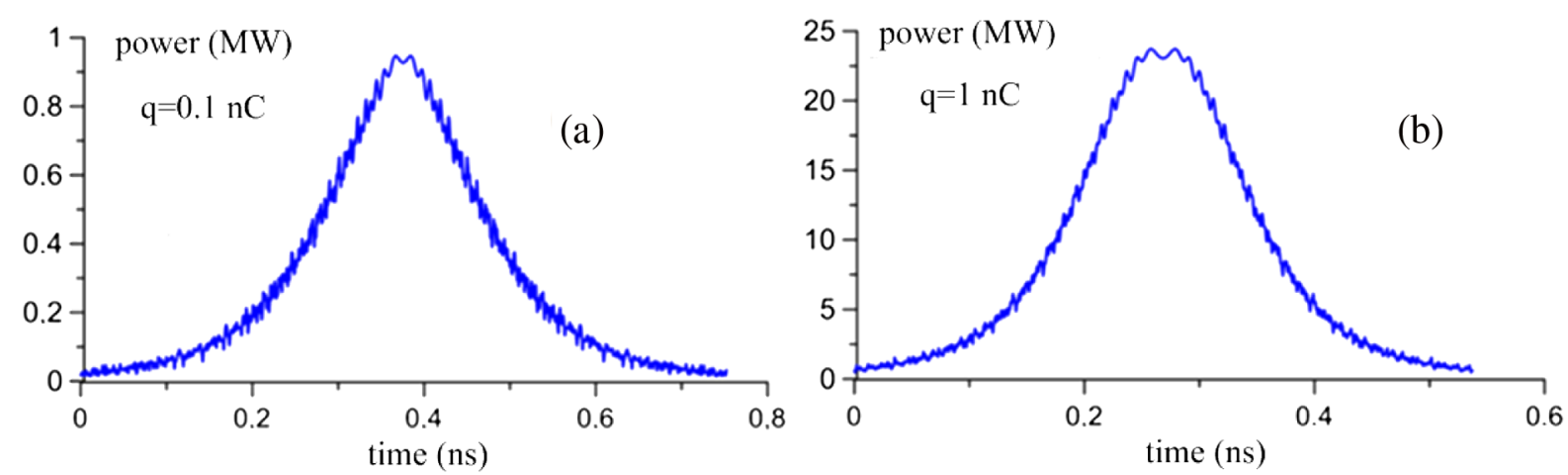

FIG. 10. Spontaneous emission from short $\left(L_{e, 0}=\lambda / 4\right)$ bunches with two different bunch charges 0.1 (a) and $1 \mathrm{nC}$ (b). The wave pulse (power versus time) radiated in the group resonance regime.

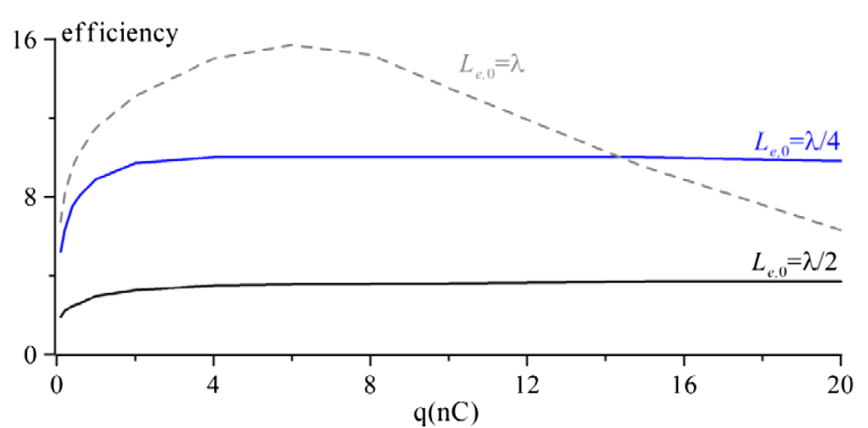

FIG. 11. The saturated efficiency versus the bunch charge at difference initial lengths of the electron bunch.

\section{DISCUSSION AND CONCLUSION}

In this paper, we have developed the theory of electron cyclotron masers to the situation when a short dense electron bunch produces spontaneous radiation of a short wave packet. This type of emission is an attractive way to realize a relatively short, simple, and efficient source of sub- $\mathrm{THz}$ radiation. The key problem here is that the spontaneous character of emission is maintained, while the phase size of the bunch with respect to the radiated wave is small enough (smaller than $<2 \pi$ ). However, even if the initial bunch is short enough, strong Coulomb fields inside the short dense bunch lead to a fast increase of the axial size of the bunch due to the Coulomb repulsion. In devices with axial character of the electron bunching in the field of the radiated wave (FELs and Cherenkov devices), this problem should be solved by means of providing either a mechanism of compensation of the Coulomb repulsion (for instance, using the negative-mass mechanism [13]) or a mechanism of axial compression of the bunch inside the radiation region. In contrast, in the case of a cyclotron maser, this problem can be solved "automatically." We show that, at definite conditions (namely, when the group wave velocity is equal to the axial electron velocity), the increase in the axial length of the bunch does not lead to an increase in the phase size of the bunch with respect to the radiated wave. In some sense, this effect (arising as a result of mutual compensation of changes in the axial and azimuthal components of the electron cyclotron phase) can be considered as an analogue of the negative-mass effect proposed in Ref. [13] for the control of the phase size of the bunch in the case of undulator radiation. However, in the case of the undulator source, the negative-mass effect decreases the phase size of the bunch just due to a decrease in the bunch length, as the Coulomb repulsion is replaced by the "Coulomb attraction." In the case of the cyclotron source, the Coulomb fields lead to repulsion of electrons, and the bunch length increases in the process of motion of the bunch through the radiation region. However, the Coulomb elongation of the bunch does not lead to an increase in its phase size.

Thus, the use of the cyclotron resonance mechanism of the emission is an attractive way for the realization of a source of powerful sub-THz pulses based on the spontaneous emission from short dense bunches. We show that a key point is the use of the regime of the group synchronism. In this situation, along with the solution of the problem of Coulomb repulsion, one more important advantage takes place; namely, the wave is emitted in the so-called superradiant regime. Since the short radiated wave packet propagates together with the electron bunch, the electron-wave interaction process is very effective and leads to the formation of a short powerful wave pulse.

Both these factors (stabilization of the phase bunch size and superradiant radiation) together provide a high efficiency achieved in the regime of spontaneous coherent radiation. The saturated efficiency in this regime is close to the efficiency achieved in the "traditional" regime of induced radiation (when the initial phase size of the bunch is not small). However, naturally, the saturation length of the induced process is significantly longer and depends on the initial signal formed by bunch edges. The additional advantage of spontaneous radiation is revealed in the cases of very great bunch charges. The results of numerical simulations shown in Fig. 11 show that an increase in the bunch charge leads to reducing the efficiency in the regime 

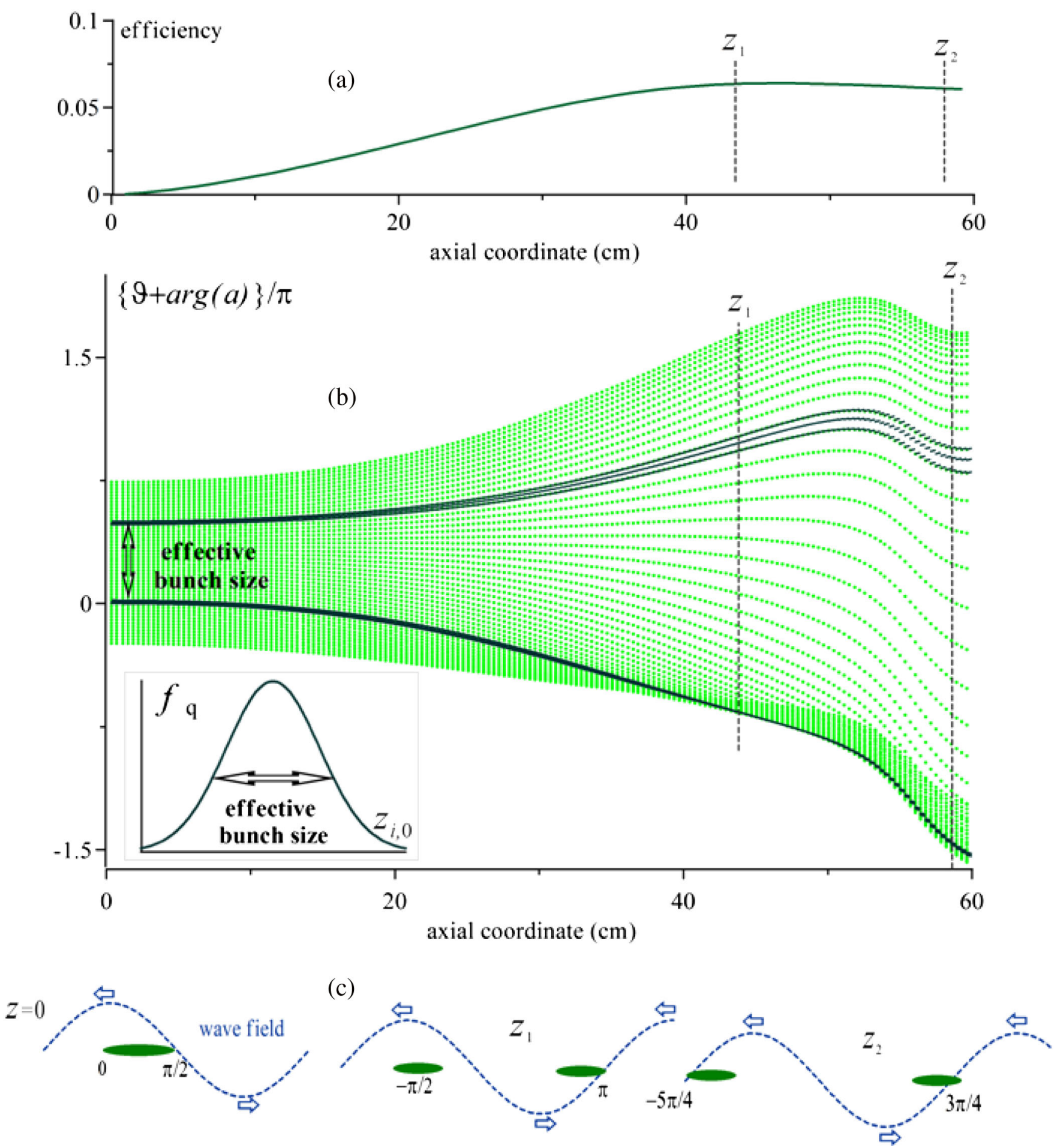

FIG. 12. (a) The efficiency of electron-wave interaction for the $0.1 \mathrm{nC}$ bunch with the initial effective length $\lambda / 4$ (the full length is $\lambda / 2$ ). (b) Distribution of electrons over their phases with respect to the wave versus the averaged axial coordinate of the bunch. (c) Schematic illustration of the dynamics of the bunch with respect to the wave phase.

of induced radiation. At the same time, if the initial phase size of the bunch is small enough to provide spontaneous radiation, the efficiency is almost independent on the bunch charge even at huge values of charges.

We have mentioned above that the Coulomb repulsion does not lead to an increase in the bunch phase size.
However, in reality, the phase size of the bunch increases; however, this is not the Coulomb fields but due to the strong interaction of electrons with the radiated wave. Figure 12 illustrate dynamics of phases of different electrons in the process of spontaneous coherent radiation in the situation when the initial effective phase size of the 
bunch is as small as $\pi / 2$. A peculiarity of the superradiant process is that in the beginning of this process the phase of the radiated wave with respect to the bunch center is $\pi / 4$ [17]. Therefore, the initial phase of the bunch front is close to zero, whereas the initial phase of the tail is close to $\pi / 2$ [Fig. 12(c)]. Since the front is placed at the maximum of the decelerating phase of the radiated rf wave, its phase decreases due to the loss of the electron energy down the nearest steady-state equilibrium (the steady-state "wave zero," $-5 \pi / 4)$. As for the tail, initially this is close to the "nonstable" zero of the wave field $(\pi / 2)$. Therefore, in the process of the electron-wave interaction, the phases of particles from the bunch tail go to the other steady-state wave zero, $-5 \pi / 4+2 \pi=3 \pi / 4$. Thus, the phase size of the bunch increases due to the electron-wave interaction. In fact, the saturation of the superradiant process corresponds to the situation when the initial bunch is divided into two bunches placed in two different steady-state "zeros" of the wave field, so that the phase distance between these bunches is close to $2 \pi$ [Fig. 12(c), $z=z_{2}$ ].

Note that such behavior of the bunch is typical only for the regime of the exact group resonance. In fact, the initial phase of the bunch with respect to the radiated wave depends on the mismatch between the wave group velocity and the axial electron velocity. In principle, it is possible to provide the situation when at the beginning of the electronwave interaction process the bunch is placed at the "proper" phase with respect to the radiated wave, such that the radiation fields provide a decrease in the phase size of the bunch. This case is not interesting from the viewpoint of the radiation process, as the efficiency in this case is relatively low. However, this case can be way to provide self-compression of the bunch by the radiated field (similar to the undulator radiation self-compression considered in Ref. [17]). We do not address this issue in this paper, hoping that it will become a subject of future papers.

\section{ACKNOWLEDGMENTS}

The work is supported by the RAS Presidium Project No. 10 (State task Project No. 0035-2018-0022) and Russian Foundation for Basic Research Project No. 1832-00351.

[1] J. G. Power, Overview of photoinjectors, AIP Conf. Proc. 1299, 20 (2010).

[2] F. Stephan et al., Detailed characterization of electron sources yielding first demonstration of european X-ray free-electron laser beam quality, Phys. Rev. ST Accel. Beams 13, 020704 (2010).

[3] J. B. Rosenzweig, A. Valloni, D. Alesini, G. Andonian, N. Bernard, L. Faillace, L. Ficcadenti, A. Fukusawa, B. Hidding, M. Migliorati, A. Mostacci, P. Musumeci, B. O. Shea, L. Palumbo, B. Spataro, and A. Yakub, Design and applications of an X-band hybrid photoinjector, Nucl. Instrum. Methods Phys. Res., Sect. A 657, 107 (2011).

[4] S. Karkare, L. Boulet, L. Cultrera, B. Dunham, X. Liu, W. Schaff, and I. Bazarov, Ultrabright and Ultrafast III-V Semiconductor Photocathodes, Phys. Rev. Lett. 112, 097601 (2014).

[5] K. J. P. Quintero, S. Antipov, A. V. Sumant, C. Jing, and S. V. Baryshev, High quantum efficiency ultrananocrystalline diamond photocathode for photoinjector applications, Appl. Phys. Lett. 105, 123103 (2014).

[6] A. Bartnik, C. Gulliford, I. Bazarov, L. Cultrera, and B. Dunham, Operational experience with nanocoulomb bunch charges in the cornell photoinjector, Phys. Rev. ST Accel. Beams 18, 083401 (2015).

[7] A. Doria, R. Bartolini, J. Feinstein, G. P. Gallerano, and R. H. Pantell, Coherent emission and gain from a bunched electron beam, IEEE J. Quantum Electron. 29, 1428 (1993).

[8] A. Gover, F. V. Hartemann, G. P. Le Sage, and N. C. Luhmann, Jr., R. S. Zhang, and C. Pellegrini, Time and Frequency Domain Analysis of Superradiant Coherent Synchrotron Radiation in a Waveguide Free-Electron Laser, Phys. Rev. Lett. 72, 1192 (1994).

[9] Y. Lurie and Y. Pinhasi, Enhanced superradiance from energy-modulated short electron bunch free-electron lasers, Phys. Rev. ST Accel. Beams 10, 080703 (2007).

[10] Y. Lurie, A. Friedman, and Y. Pinhasi, Single pass, THz spectral range free-electron laser driven by a photocathode hybrid rf linear accelerator, Phys. Rev. ST Accel. Beams 18, 070701 (2015).

[11] V. L. Bratman, D. A. Jaroszynsky, S. V. Samsonov, and A. V. Savilov, Generation of ultrashort quasiunipolar electromagnetic pulses from quasiplanar electron bunches, Nucl. Instrum. Methods Phys. Res., Sect. A 475, 436 (2001).

[12] K. Lee, J. Mun, S. H. Park, R.-H. Jang, Y. U. Jeong, and N. A. Vinokurov, Numerical investigation of the radiation characteristics of a variable-period helical undulator, Nucl. Instrum. Methods Phys. Res., Sect. A 776, 27 (2015).

[13] N. Balal, I. V. Bandurkin, V. L. Bratman, E. Magory, and A. V. Savilov, Negative-mass mitigation of coulomb repulsion for terahertz undulator radiation of electron bunches, Appl. Phys. Lett. 107, 163505 (2015).

[14] Y. Lurie, V. L. Bratman, and A. V. Savilov, Energy enhancement and spectrum narrowing in terahertz electron sources due to negative mass instability, Phys. Rev. Accel. Beams 19, 050704 (2016).

[15] V. L. Bratman, N. S. Ginzburg, and M. I. Petelin, Common properties of free electron lasers, Opt. Commun. 30, 409 (1979).

[16] E. Curry, S. Fabbri, P. Musumeci, and A. Gover, THzDriven Zero-Slippage IFEL scheme for phase space manipulation, New J. Phys. 18, 113045 (2016).

[17] I. V. Bandurkin, Yu. S. Oparina, and A. V. Savilov, Superradiative self-compression of photo-injector electron bunches, Appl. Phys. Lett. 110, 263508 (2017).

[18] I. V. Bandurkin, I. S. Kurakin, and A. V. Savilov, Compression of a photoinjector electron bunch in the negativemass undulator, Phys. Rev. Accel. Beams 20, 020704 (2017). 
[19] M. I. Petelin, On the theory of ultrarelativistic cyclotron self-resonance masers, Radiophys. Quantum Electron. 17, 686 (1974).

[20] P. Sprangle and W. M. Manheimer, Coherent nonlinear theory of a cyclotron instability, Phys. Fluids 18, 224 (1975).

[21] P. Sprangle and A. T. Drobot, The linear and self-consistent nonlinear theory of the electron cyclotron maser instability, IEEE Trans. Microwave Theory Tech. 25, 528 (1977).

[22] V. L. Bratman, N. S. Ginzburg, G. S. Nusinovich, M. I. Petelin, and P.S. Strelkov, Relativistic gyrotrons and cyclotron autoresonance masers, Int. J. Electron. 51, 541 (1981).

[23] V. L. Bratman, G. G. Denisov, N. S. Ginzburg, and M. I. Petelin, FEL's with bragg reflection resonators: cyclotron autoresonance masers versus ubitrons, IEEE J. Quantum Electron. 19, 282 (1983).

[24] A. V. Savilov, Cyclotron resonance maser with a tapered magnetic field in the regime of "Nonresonant" trapping of the electron beam, Phys. Rev. E 64, 066501 (2001).
[25] N. S. Ginzburg, I. V. Zotova, and A. S. Sergeev, Cyclotron superradiance of a moving electron swarm under group synchronization conditions, JETP Lett. 60, 513 (1994).

[26] N. S. Ginzburg, A. S. Sergeev, I. V. Konoplev, I. V. Zotova, A. D. R. Phelps, A. W. Cross, S. Cook, P. Aitken, V. G. Shpak, M. I. Yalandin, and S. A. Shunailov, Experimental Observation of Cyclotron Superradiance under Group Synchronism Conditions, Phys. Rev. Lett. 78, 2365 (1997).

[27] I. V. Bandurkin, I. V. Osharin, and A. V. Savilov, Cyclotron radiation cooling of a short electron bunch kicked in an undulator with guiding magnetic field, Phys. Rev. ST Accel. Beams 18, 110702 (2015).

[28] R. D. Richtmyer, Principles of Advanced Mathematical Physics (Springer-Verlag, Berlin, 1978), Vol. 1.

[29] A. Friedman, N. Balal, E. Dyunin, Yu. Lurie, E. Magori, V. L. Bratman, J. Rosenzweig, and H. Lay, and A. Gover, Configuration and status of the Israeli $\mathrm{THz}$ free electron laser, in Proceedings of the 36th International Free Electron Laser Conference, Basel, Switzerland, 2014 (JACoW, 2014), pp. 553-555. 